\title{
Teaching Research on the manual Model of Product Design
}

\author{
Liu Ying ${ }^{1}$, Liu Xiaojing ${ }^{1}$ and Chen $\mathrm{Yi}^{2}$, a \\ ${ }^{1}$ Cnetral South University, Industrial Design Department, 410083 Changsha Hunan, China \\ ${ }^{2}$ Guzhi Science and Technology Co., Ltd. 410083 Changsha Hunan, China
}

\begin{abstract}
This paper discribes the spatial thinking ability ,materials processing ,design easthetics application and technical implementation are four aspects that very important to cultivate students' professional quality when building a manual model. It stresses the handwork 1 model course has played positive significance in the aspect of design basic teaching under the background of High-Tech.
\end{abstract}

\section{Introduction}

In current teaching of Industrial Design major, the technical methods of model production has become abundant. The computer auxiliary manufacture and 3D printing technology can present the design effects in a rapid and accurate manner ${ }^{[1]}$. There numbers of softwares that can produce 3D spatial effect. However, its virtual effect has the 2D plane display feature, and the computer has certain limitations when shaped displaying (perspective view, chromatic aberration of layers, depth of field of camera etc.). In addition, the rapid processing technology directly generates the substantial results, and the teaching process can skip the process of design implementation ${ }^{[2]}$. It can be seen from the essence of experience-typed teaching, and the convenient technical methods cannot replace the process of direct experience accumulation completely. However, the process of handmade model can display or represent the design content through creating the actual space scene or repeating experience ${ }^{[3]}$.Students can understand and construct knowledge, develop practical ability and generate design significance in the course of experiencing the deduction process.

\section{Cultivation of professional ability}

The handmade model is a form of 3D direct visual expression, which is the effective method of understanding the virtual space indention of product in the course of shaped design derivation ${ }^{[4]}$. The production process includes several steps,for example, interpret drawings, identify drawings, spatial thinking, shape production, design elaboration, surface treatment. Each production step has different focus on the professional training of students. As a whole, manual model making is a practical training to cultivate students' spatial imagination ability, and to exercise students' ability to solve design problems according to the actual situation.

\subsection{Imagination thinking methods}

Designing drawings is the basis for model production and it is the special "Visual Language" of designers ${ }^{[5]}$. Model production firstly requires the students to learn how to interpret drawings and identify drawing, recognize the marks, labels and rules of graphical representation, understand various graphic symbols as the designing language, as well as master the meaning of various designing elements. The purpose of interpreting drawings and identifying drawing is to construct the 2Dtyped graphical description as the virtual 3D spatial in mind. The process is the result of mutual penetration operation and mutual deepened complementation of two kinds of imagination thinking methods.

The handmade model is a converting process of designing concept from 2D graphic representations to 3D substantial space. It enables students to present designing thinking from $3 \mathrm{D}$ visual field and sense the stereoscopic space relationship. It reflect the complex spatial problems which cannot be understood in the plane drawings. Beginners are not good at space analysis and shaping they usually be constrained to the 2D plane concepts on the draft designing step of graphical analysis and shape treatment with short thinking on the structure, organization and composition in space. Decompose the spatial structure by modeling, make use of internal and external consistency of space as well as the penetration of space to generate the medium for participating space and experiencing designing elements.

Through the visual observation and sensation to the handmade model, Students can understand the relationship between structure and surface, part and whole, as well as unit and environment. The whole process enables students to directly feel the physical dimension and the sense of space ${ }^{[6]}$. Comparing the spatial conditions with the graphical conditions, and the various emerging problems on substantial shapes can

* Corresponding author: ${ }^{a}$ 48316365@qq.com 
strengthen the controlling ability of students to the spatial shape and ratio scale from $3 \mathrm{D}$ visual field.

\subsection{Knowledge of materials}

Through the handmade model, Students can directly learn some relevant knowledge on the structural properties and shaping process of model materials etc ${ }^{[7]}$. Understanding the properties of materials is the basis of designing, Otherness of products' function and shapes determines their diversity, process involved in the model production. Teachers have to help students to improve basic cognition on materials technology through theory lectures and demonstrations,so, students can gradually accumulate knowledge about properties of materials and experiences of processing technology.

Except physical properties, product design also emphasize the sensation of materials. The texture property of materials and expression of beauty sense are realized in the materials selection and visual configuration. Many sorts of textures that formed by architectual difference of material show characteristics of diferrent texture. The perceptual system of human beings obtain the perceptual information from the surface texture properties of model so as to generate the experienced psychological comprehensive senses: soft and hard, light and weight, cold and warm, dry and wet, thick and thin etc. The material, texture and physical properties etc. for the materials of model are all the conditions that need to be considered by the students when selecting the materials of model ${ }^{[8]}$.

\subsection{Aesthetic experience}

The manual model is the miniature reflection of the design content. It represents the design concept with the elements of the entity shape, the structure line, the volume relation and so on, and needs to conform to the law of the design form beauty. The basis of formal beauty is a sense of balance between vision and psychology, which comes from the balance of form, colour and space. Students must abide by the rules of proportion and scale, rhythm and rhythm, contrast and unity in the process of manual model making. The structure lines, the outline lines and the parting lines need to be strengthened in different degrees. The functional view plane, transition surface and subtraction surface also require the variation of space rhythm. In addition, the training of colour in manual model making has higher requirements than graphic design. The colour processing of the model is not only the generalization of the design effect, but also the colour change factor under the condition of space light. The intensity, angle, distance and environment of the light source will have a direct impact on the colour performance of the model, even with the original design effect diagram to produce the colour difference.

From the visual performance point of view, the manual model should not only reflect the design content intuitively and objectively, The application of aesthetics and the treatment of art can enrich the students aesthetic experience and enhance the theoretical learning effect of design aesthetics. This kind of aesthetics is very important for the design works to have artistic appeal

\subsection{Craft skills}

Skilled process is the guarantee of model making implementation. Students need to be trained in the relevant knowledge and be able to master various process and tool operation skills. Manual model making process can intuitively show the process of processing operation. Students can experience the characteristics of various tools by hand operation and understand the specific use method. In view of some exploratory design, the performance of some products, such as strength, rigidity, vibration and noise, needs to be proved repeatedly. Students need to analyze and calculate the manual model to get the relevant data of product structure and performance, and then apply the results of theoretical analysis and size structure to the improvement of the model. The students can verify the rationality of theoretical analysis by handwork model making, and provide the feasibility basis for the model to the actual production transformation of the product.

Based on the technical implementation of each process, students can express the design ideas more freely, consolidate the professional theory knowledge, and solidify the basis for the design practice work in the future.

As for the technical implementation of each production step, the students are able to freely express their own designing concepts, strengthen the professional theoretic knowledge, as well as reinforce the foundation for the practical design work in the future.

\section{Conclusion}

Basing on the above analysis,by handwork model making, students can understand the form connection of each interface in the space in an all-round way, and train the students to look at the design content in a systematic way, so as to avoid forming one-sided and isolated design thinking formula. From the abstract form to the two-dimensional scheme presupposition to the threedimensional entity model, with the three-dimensional deepening of the graphic thinking, Manual model making provides an experience-intuitive experience process for students to design ideas, and an effective teaching method for college design education to break through the teaching cycle of from picture to picture.

\section{Acknowledgment}

The paper is the final supporting material for Hunan philosophy and social science foundation project "Industrial design value and performance evaluation research", Item No.: 16yba369 


\section{References}

1. Gong Wenfei, Yu Huilan, Improve Practical Ability Starts from Model Production-Research on Teaching of Model Production for Students in Industrial Design Major [J]. Changchun University of Science and Technology Academic Journal (High-education), 04 (2007)

2. Bao Lei. Analysis on Substatial Model Design and Production of Industrial Design. Nanjing University of the Arts Academic Journal (Arts and Design), 02 (2008)

3. Liander Caney. Spirit of Apple Design: Johnny•Ive. Times Press, 09 (2014)

4. Liu Ming. Research on Practical Teaching of Product Design Major. Teaching Practice and Exploration, 06 (2016).

5. Liu Guanzhong, Design is an indestructible "The Third Wisdom"of Human Beings in Future [J] Design, 12 (2013).

6. Lin Jiaxin, Wang Huabing etc. Current Status and Exploration of Industrial Design ABS Model Production Teaching Experience.Tu Xue Academic Journal, 07 (2013)

7. Zhao Jianghong. Meaning of Design Arts.Hunan University Press, 04 (2005).

8. Yao Limin, Duan Wenyu. Analysis on Teaching Method Reform in High Education.China University Teaching, 08 (2013)

9. Wu Qiong. Materials and Process Analysis on Product Handmade Model. Package Engineering, 29 (2008.)

10. $\mathrm{Wu}$ Qiong. Draft Model and Product Design.Mechanical Design, 23 (2006). 